\title{
La complexité de la prise en charge odontostomatologique de la dysplasie cléido-crânienne (DCC) : rapport de cas et revue de littérature
}

\author{
Khadija Moukram ${ }^{1}$, Olaya Medaghri Alaoui ${ }^{1}$, A. Tahiri ${ }^{2}$, Ihssan BenYahya ${ }^{1}$ \\ ${ }^{1}$ Service d'odontologie chirurgicale, Centre de consultation et de traitement dentaire, CHU, Casablanca, Maroc \\ ${ }^{2}$ Médecin dentiste exerçant en secteur privé, Casablanca, Maroc
}

(Reçu le 24 mars 2016, accepté le 26 août 2016)

Mots clés :

dysplasie

cléido-crânienne /

dents incluses /

dents surnuméraires /

CBFA1/RUNX2
Key words:

cleido cranial

dysplasia / impacted

teeth / supernumerary

teeth / CBFA1/RUNX2

\begin{abstract}
Résumé - Introduction : La dysplasie cléido-crânienne (DCC), anciennement connue sous le nom de dysostose cléidocrânienne, est une maladie génétique rare, aux formes d'expressions très variables et inconstantes. Observations : Un cas clinique pris en charge en collaboration entre le service d'odontologie chirurgicale et d'orthodontie du centre de consultation et traitement dentaire de Casablanca illustre notre propos. Il s'agissait d'un patient de 22 ans, consultant pour un retard d'éruption des incisives supérieures droites. À partir des éléments cliniques et radiologiques relevés lors des examens initiaux (atteinte claviculaire, anomalies dentaires et déformations cranio-faciales), le diagnostic évoqué était une dysplasie cléido-crânienne. La prise en charge du patient consistait à corriger les anomalies dentaires, à extraire les dents surnuméraires et à positionner les dents permanentes sur l'arcade de manière à obtenir une occlusion fonctionnelle, durable et esthétique. Discussion : La dysplasie cléido-crânienne (DCC) se manifeste principalement par des anomalies squelettiques, claviculaires, et odontostomatologiques nombreuses et évolutives. Ces dernières restent encore souvent à l'origine du diagnostic précoce et se révèlent parmi les plus handicapantes pour les patients. Ainsi, la prise en charge s'axe presque exclusivement sur la sphère oro-faciale et mobilise l'ensemble des disciplines odontologiques. Aujourd'hui, les démarches thérapeutiques tiennent compte des mécanismes physiopathologiques de l'affection et aboutissent à des résultats satisfaisants justifiant de longues périodes de traitement mobilisant les spécialistes et le patient. Conclusion : Dans tous les cas, c'est une véritable collaboration multidisciplinaire qui se met en place entre le médecin, le chirurgien-dentiste, le chirurgien maxillofacial et l'orthodontiste pour corriger l'hypoplasie du massif facial et les anomalies dentaires.
\end{abstract}

\begin{abstract}
The complexity of the management of cleidocranial dysplasia (CCD) in dentistry and oral surgery: case report and literature review. Introduction: Cleidocranial dysplasia (CCD), previously known as cleidocranial dysostosis, is a rare genetic disease, with highly variable and inconsistent clinical forms. Observations: A case report, treated in collaboration between the surgical and orthodontic departments of the Center of Consultation and Dental Treatment of Casablanca, illustrates this subject. A 22-year-old male patient complaining of delayed eruption of the upper right incisors was referred to the department of oral surgery of this center. Clinical and radiographic information gathered from this examination (a clavicular defect, dental abnormalities and craniofacial anomalies) suggested a diagnosis of cleidocranial dysplasia. The dental care objectives were to correct the dental abnormalities, extract the supernumerary teeth and correct the position of the permanent teeth to obtain a functional, lasting and esthetic occlusion. Discussion: CCD causes various skeletal, clavicular and odontostomatological abnormalities. The dental signs of cleidocranial dysplasia (CCD) often lead to early diagnosis; thus, the treatment is mainly centered on the orofacial sphere, and concerns all dental disciplines. Nowadays, therapeutic strategies are based on the pathophysiology of this condition and mostly lead to good results, justifying the long treatment periods, affecting both patients and specialists. Conclusion: A multidisciplinary approach involving the generalist, the oral surgeon, the maxillofacial surgeon and the orthodontist is absolutely necessary to correct the hypoplasia of the facial skeleton and the dental abnormalities in cleidocranial dysplasia.
\end{abstract}

\footnotetext{
* Correspondance : khadijamoukram0@gmail.com
}

This is an Open Access article distributed under the terms of the Creative Commons Attribution License (http://creativecommons.org/licenses/by/4.0), which permits unrestricted use, distribution, and reproduction in any medium, provided the original work is properly cited 


\section{Introduction}

La dysplasie ou dysostose cléido-crânienne est étymologiquement issue du grec ancien : Dys = mauvais, Osteon $=0$, Kleis $=$ clavicule, Kranion = crâne. Une dysplasie désigne toute anomalie du développement d'une cellule, d'un tissu ou d'un organe se traduisant par une déformation ou une malformation cellulaire, tissulaire ou organique. Cependant, la dysostose désigne une déformation qui touche préférentiellement une ou plusieurs pièces osseuses. De ce fait, depuis 1970, on parle de « dysplasie » plutôt que de « dysostose » qui est un terme plus restrictif [1].

La DCC est une maladie génétique rare, aux formes d'expressions variables. Les deux sexes sont indifféremment affectés par le syndrome sans qu'il existe de prédominance dans une quelconque population humaine. Le syndrome affecterait un individu sur un million, mais l'ensemble des auteurs s'accordent sur ce point : les approches statistiques du syndrome sont approximatives et la prévalence est sans aucun doute considérablement sous-évaluée [2]. Il s'agit d'une maladie, peu fréquente et évolutive, qui se manifeste principalement par des anomalies squelettiques, claviculaires, et craniofaciales nombreuses et évolutives. Parmi elles, les signes dentaires restent encore souvent à l'origine du diagnostic et se révèlent parmi les plus handicapants pour les patients [2].

Contrairement à d'autres syndromes associant des anomalies squelettiques et dentaires, le tableau clinique de DCC est peu connu. Par conséquent, ce travail vise à illustrer la prise en charge bucco-dentaire de ces patients, qui implique une collaboration pluridisciplinaire entre le chirurgien oral, le chirurgien maxillo-facial et l'orthodontiste [3].

\section{Cas clinique}

Un patient, âgé de 22 ans, sans antécédent médicochirurgical particulier, se présentait en consultation pour un retard d'éruption des incisives supérieures droites. Le patient présentait un développement staturo-pondéral légèrement subnormal:

- les mains étaient courtes et trapues, les pouces étaient raccourcis (Fig. 1a) ;

- les épaules étaient tombantes et projetées en avant, la musculature bien développée avec un thorax étroit (Fig. 1b).

L'examen maxillo-facial révélait des bosses frontales proéminentes avec un massif crânien plus développé dans le sens transversal que dans le sens sagittal (brachycéphalie). Le faciès était triangulaire et plat, les pommettes effacées, et le nez était épaté avec une racine élargie et une cloison légèrement déviée. Une éversion de la lèvre inférieure par rapport à la lèvre supérieure qui était plus fine a été également notée (Fig. 2a). Le profil sous-nasal était concave et le menton proéminent. La vue de profil révélait une légère pseudo-exophtalmie, et une

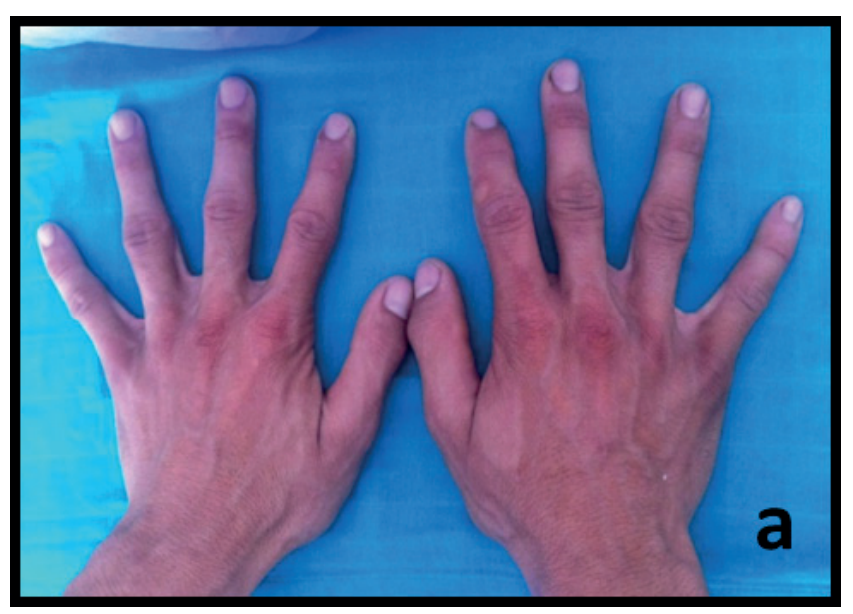

Fig. 1a. Mains courtes.

Fig. 1a. Short hands.

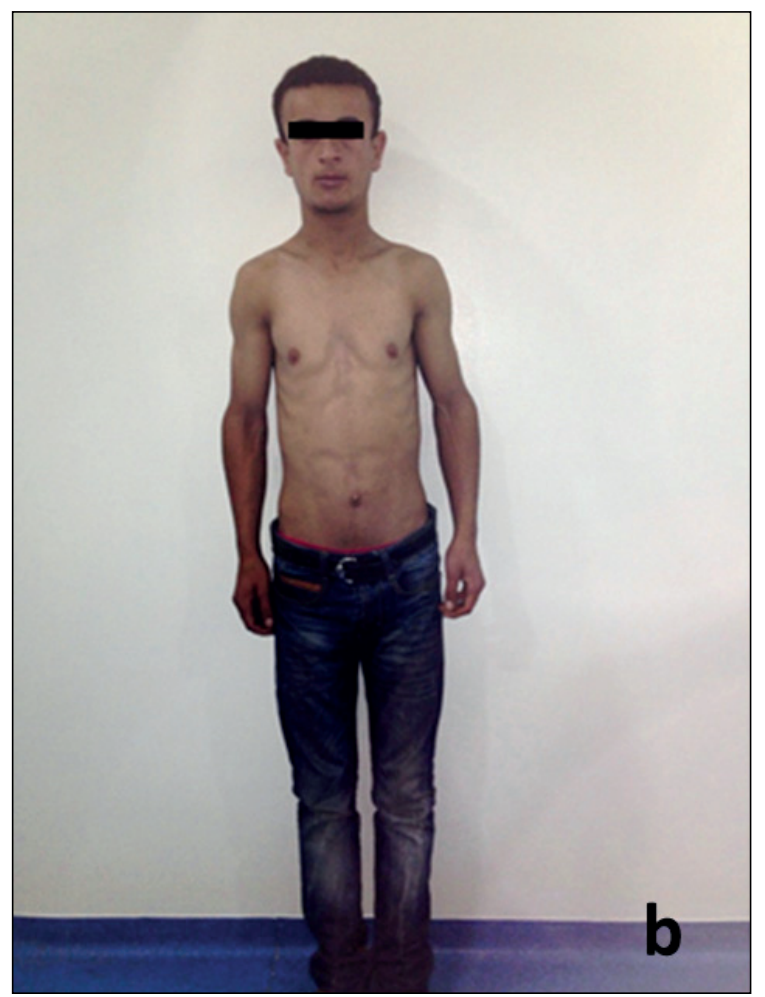

Fig. 1b. Posture du patient : épaules tombantes, un thorax étroit et des membres courts.

Fig. 1b. Patient's posture: sloping shoulders, narrow thorax and short members.

ensellure nasale très marquée (Fig. 2b). L'examen de la cavité orale révélait une endognathie maxillaire droite, un palais étroit et profond, la persistance des canines temporaires maxillaires, la rotation de la 43 et un désalignement des dents sur les deux arcades. 


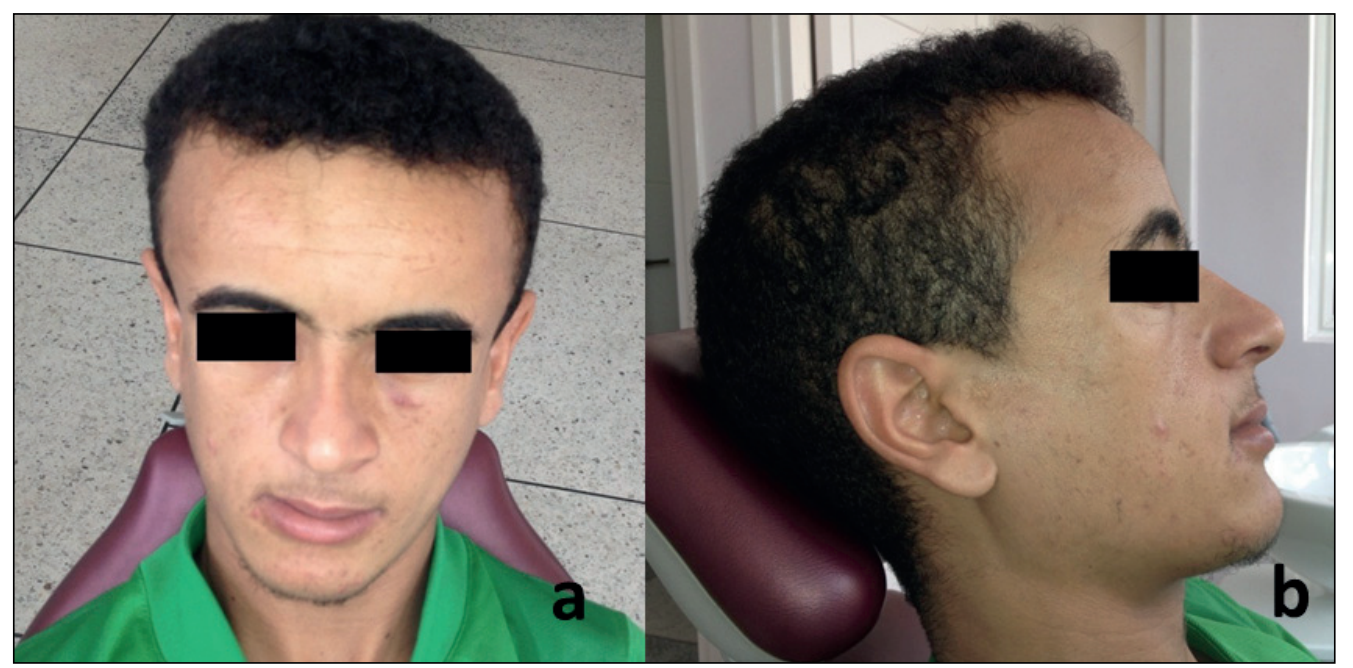

Fig. 2a, b. Dysmorphie craniofaciale. Fig. 2a, b. Craniofacial dysmorphia.

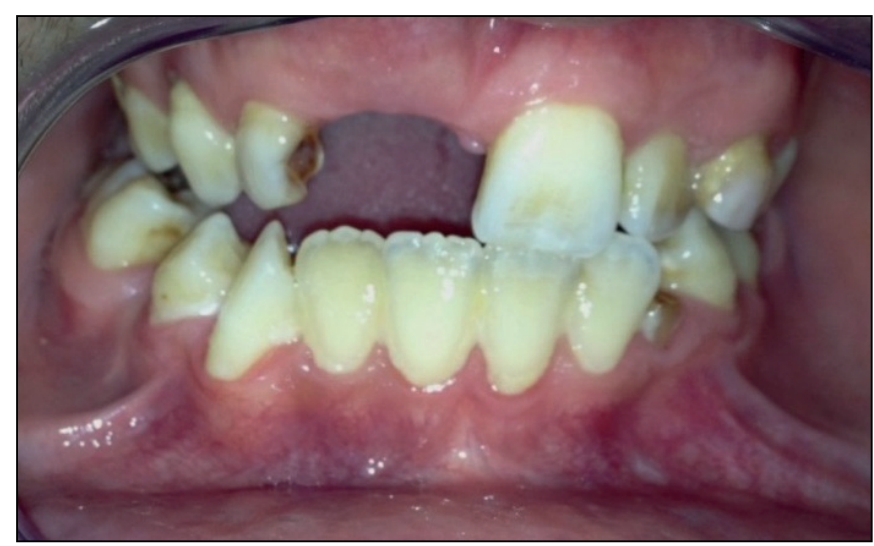

Fig. 3. Vue intra-orale des dents maxillaires et mandibulaires en occlusion montrant l'absence des incisives supérieures droites. Fig. 3. Intraoral view of maxillary and mandibular teeth in occlusion showing the absence of the upper right incisors.

Le patient présentait une hygiène insuffisante avec accumulation de plaque (Fig. 3).

La radiographie panoramique initiale révélait (Fig. 4) :

- sur le plan osseux :

- des sinus maxillaires peu développés,

- des arcades zygomatiques fines,

- des processus coronoïdes fins,

- des bords des ramus ascendants parallèles ;

- sur le plan dentaire :

- des axes dentaires anarchiques (mésio ou distoversion, rotation),

- la persistance des deux canines temporaires maxillaires,

- un odontome en regard des incisives supérieures droites,

- de nombreuses dents incluses.

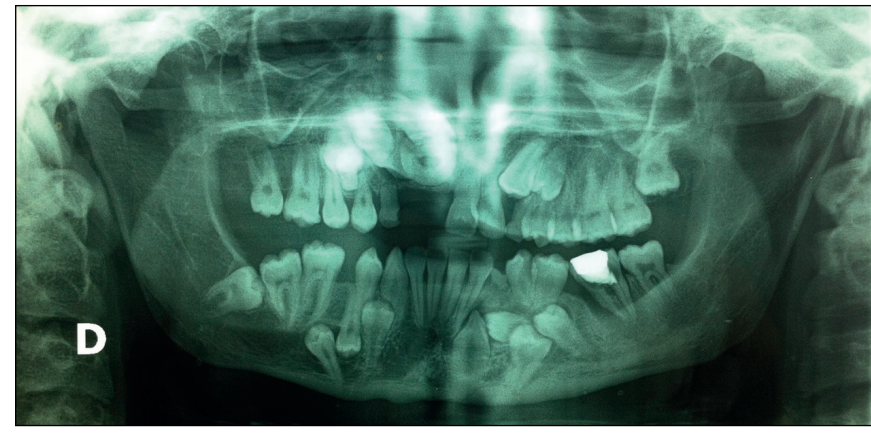

Fig. 4. Radiographie panoramique. Une polyinclusion à l'origine d'un encombrement intra-osseux important.

Fig. 4. Panoramic radiography. The multiple inclusion, creating an important intraosseous lack of space.

La téléradiographie de profil du patient permettait d'apprécier l'ensemble des signes suivants : des bosses frontales proéminentes, une béance de la fontanelle bregmatique, l'absence de l'os nasal, un pseudo-prognathisme mandibulaire, un angle mandibulaire ouvert et de nombreuses dents surnuméraires incluses (Fig. 5).

Afin d'affiner le diagnostic, un examen Cone Beam a été réalisé pour estimer le nombre de dents incluses et préciser les situations spatiales des dents retenues ainsi que leurs rapports avec les structures avoisinantes, notamment les structures critiques tel le nerf alvéolaire inférieur. Cet examen a été non seulement un moyen diagnostique précis, mais aussi un support dans la planification des interventions chirurgicales (Fig. 6).

Sur la radiographie du thorax : celui-ci était étroit, en forme de cône, avec des côtes obliques vers le bas. Une hypoplasie bilatérale du $1 / 3$ moyen et du $1 / 3$ médian (sternal) de la clavicule était également visible (Fig. 7). 


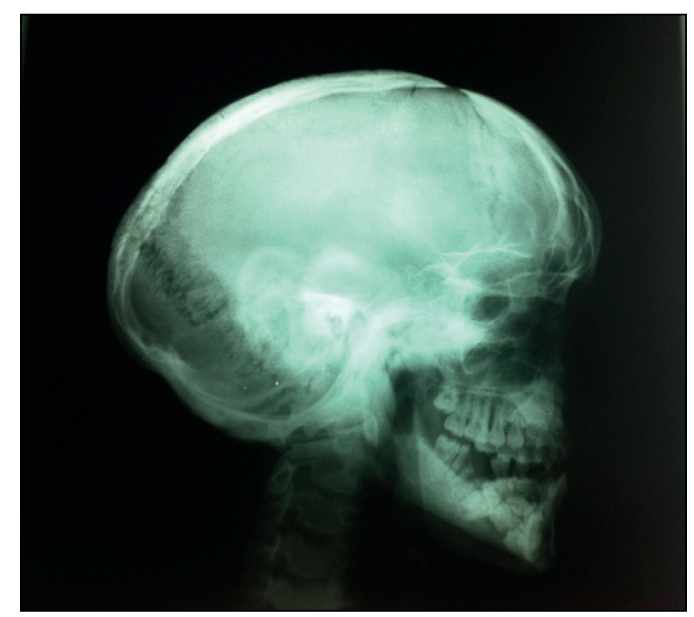

Fig. 5. Téléradiographie de profil montrant des bosses frontales proéminentes, une béance de la fontanelle bregmatique, l'absence de l'os nasal, un pseudo-prognathisme mandibulaire, un angle mandibulaire ouvert, et de nombreuses dents surnuméraires et incluses.

Fig. 5. Profile teleradiography showing a frontal bossing, an open bregmatic fontanelle, the absence of the nasal bone, a pseudo-mandibular prognathism, an open mandibular angle, and many supernumerary and impacted teeth.

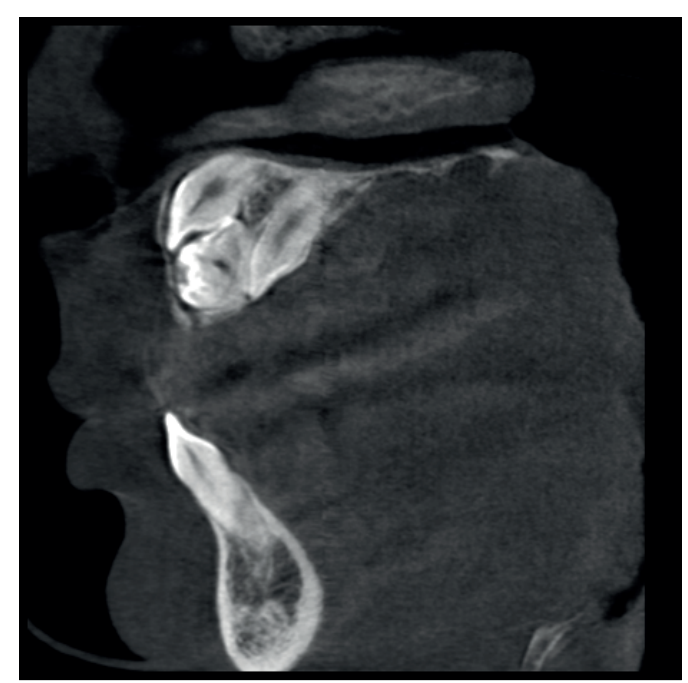

Fig. 6. Coupe sagittale montrant les rapports de l'odontome avec la 11 et la 12 et les structures avoisinantes.

Fig. 6. Sagittal section showing the relationships of the odontome with 11 and 12 and surrounding structures.

À partir des éléments cliniques et radiographiques relevés lors de ces examens (atteinte claviculaire, anomalies dentaires et déformations cranio-faciales), le diagnostic évoqué était une dysplasie cléido-crânienne.

Il est à noter que les parents et la fratrie de ce patient étaient indemnes, ce qui laisse penser à une forme sporadique de la maladie.

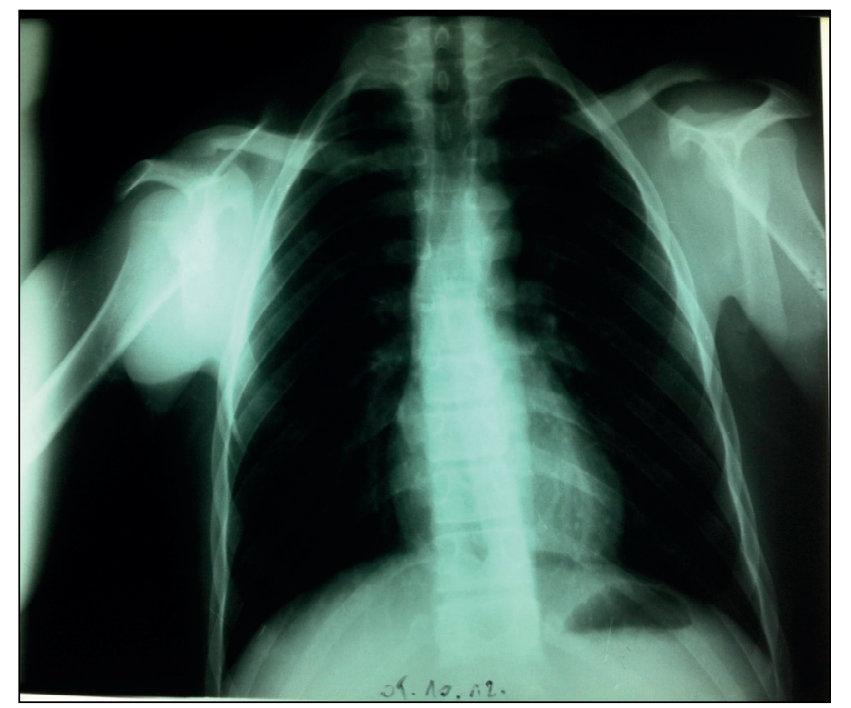

Fig. 7. Radiographie thoracique de face montrant un thorax étroit, des côtes obliques et des clavicules hypoplasiques.

Fig. 7. Frontal thoracic radiography showing a narrow thorax, wavy ribs and hypoplastic clavicles.

À la suite de ce constat, et après concertation interdisciplinaire avec nos confrères orthodontistes, et en raison à la fois de la présence sur l'arcade de dents temporaires, d'un nombre considérable de dents incluses et de l'âge du patient, une prise en charge chirurgico-orthodontique était décidée.

Le premier temps consistait en l'avulsion de toutes les dents temporaires, surnuméraires, des dents de sagesse et de l'odontome, associée au dégagement et collage de dispositifs de traction orthodontique sur les dents incluses et retenues afin de les diriger sur l'arcade de manière à obtenir une occlusion fonctionnelle, durable et esthétique, et ceci en huit temps interventionnels distincts.

Dans un second temps, le patient sera soumis à une réhabilitation prothétique afin de combler les édentations partielles persistantes.

Les techniques d'hygiène ont été maîtrisées avant que le contrôle de plaque ne soit altéré par la présence prolongée des appareillages orthodontiques.

- La $1^{\text {re }}$ intervention : extraction des racines résiduelles de la 73 , de la 33 et de la 37.

- La $2^{\mathrm{e}}$ intervention : extraction chirurgicale de la 28 suivie de l'avulsion de la 63.

- La $3^{\mathrm{e}}$ intervention : extraction chirurgicale de la 48.

- La $4^{\mathrm{e}}$ intervention: dégagement chirurgical des 2 prémolaires supérieures gauches incluses ( 24 bis, 25 bis) suivi d'un collage des attaches (boutons/brackets) afin d'effectuer la traction orthodontique.

- La $5^{\mathrm{e}}$ intervention : extraction de la 34 et avulsion chirurgicale des prémolaires surnuméraires incluses du secteur mandibulaire gauche. 


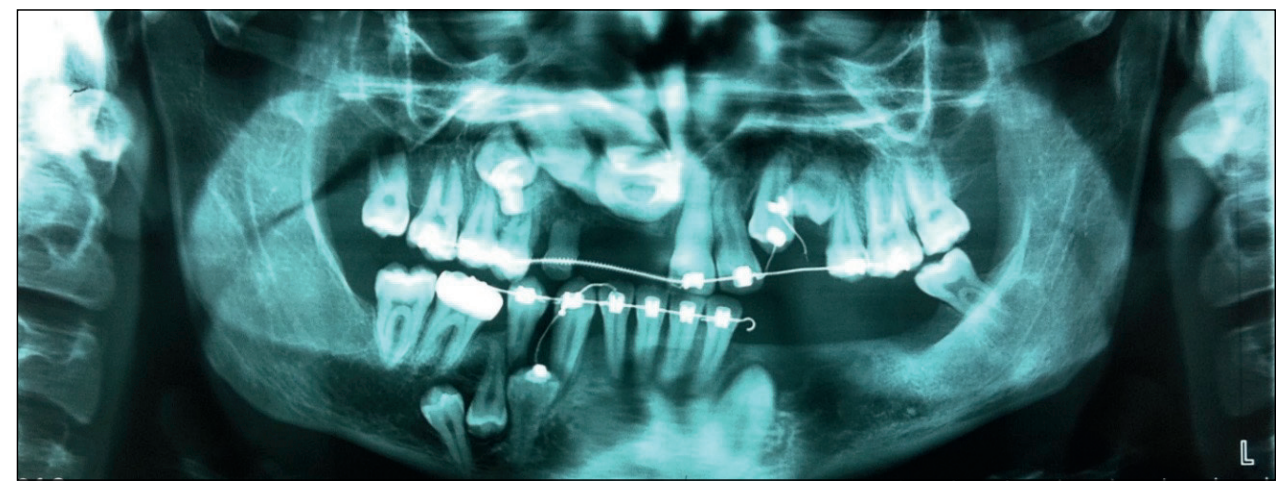

Fig. 8. Radiographie panoramique de contrôle après trois ans de traitement : une petite évolution de la 24 qui est toujours en traction et en sous-occlusion; une difficulté d'évolution de la 44 (présence de deux dents surnuméraires 44 bis et 45 bis); la 25 toujours en traction. Fig. 8. Control panoramic radiography three years post treatment: a small evolution in 24 which is always undergoing orthodontic traction, and under occlusion; a difficulty in the evolution of the 44 (presence of two supernumerary teeth $44 a$ and $45 a$ ); the 25 is always under orthodontic traction.
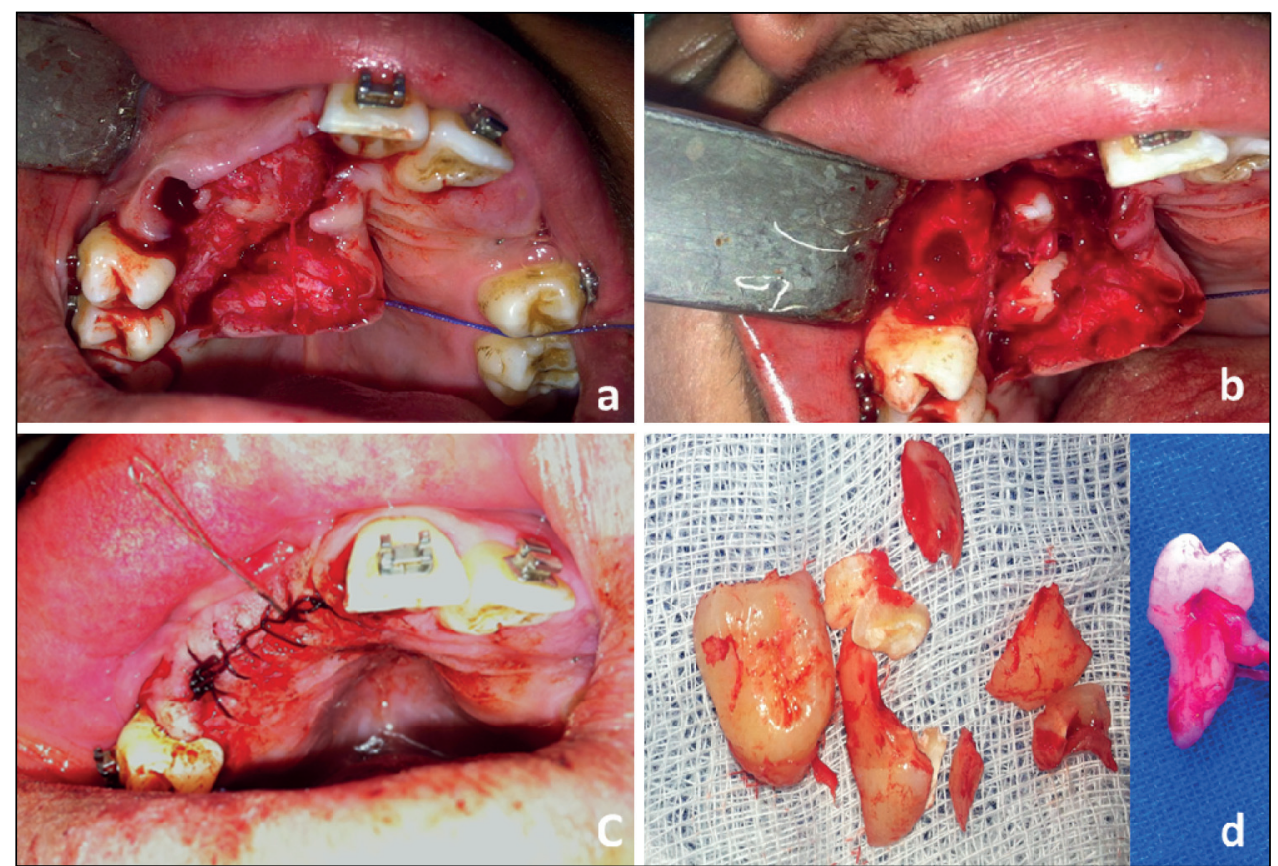

Fig. 9. Photographies de l'intervention $n^{\circ} 7$.

a : Dégagement osseux de la tumeur. b : Aspect de la cavité tumorale. c : Collage du dispositif et sutures. $d$ : Dents miniatures et prémolaire extraites.

Fig. 9. The seventh intervention pictures.

a: Bone exposure of the tumor. b: Aspect of the tumor cavity. c: Gluing of the orthodontic devices and sutures. d: Miniature teeth and premolar (14 bis) extracted.

- La $6^{\mathrm{e}}$ intervention : dégagement chirurgical de la 44 et collage d'une attache (Fig. 8).

- La $7^{\mathrm{e}}$ intervention comprenait (Figs. 9 a, b, c, d) :

- l'exérèse de l'odontome en regard du secteur antéro-supérieur droit ;

- l'extraction chirurgicale de la prémolaire surnuméraire du même secteur (14 bis) ;
- le dégagement chirurgical de la 12 accompagné du collage d'une attache (brackets).

- L'intervention $n^{\circ} 8$ comprenait :

- l'extraction chirurgicale des prémolaires surnuméraires (44 bis, 45 bis) incluses ;

- l'extraction de la 43 qui était totalement dénuée d'os en vestibulaire. 

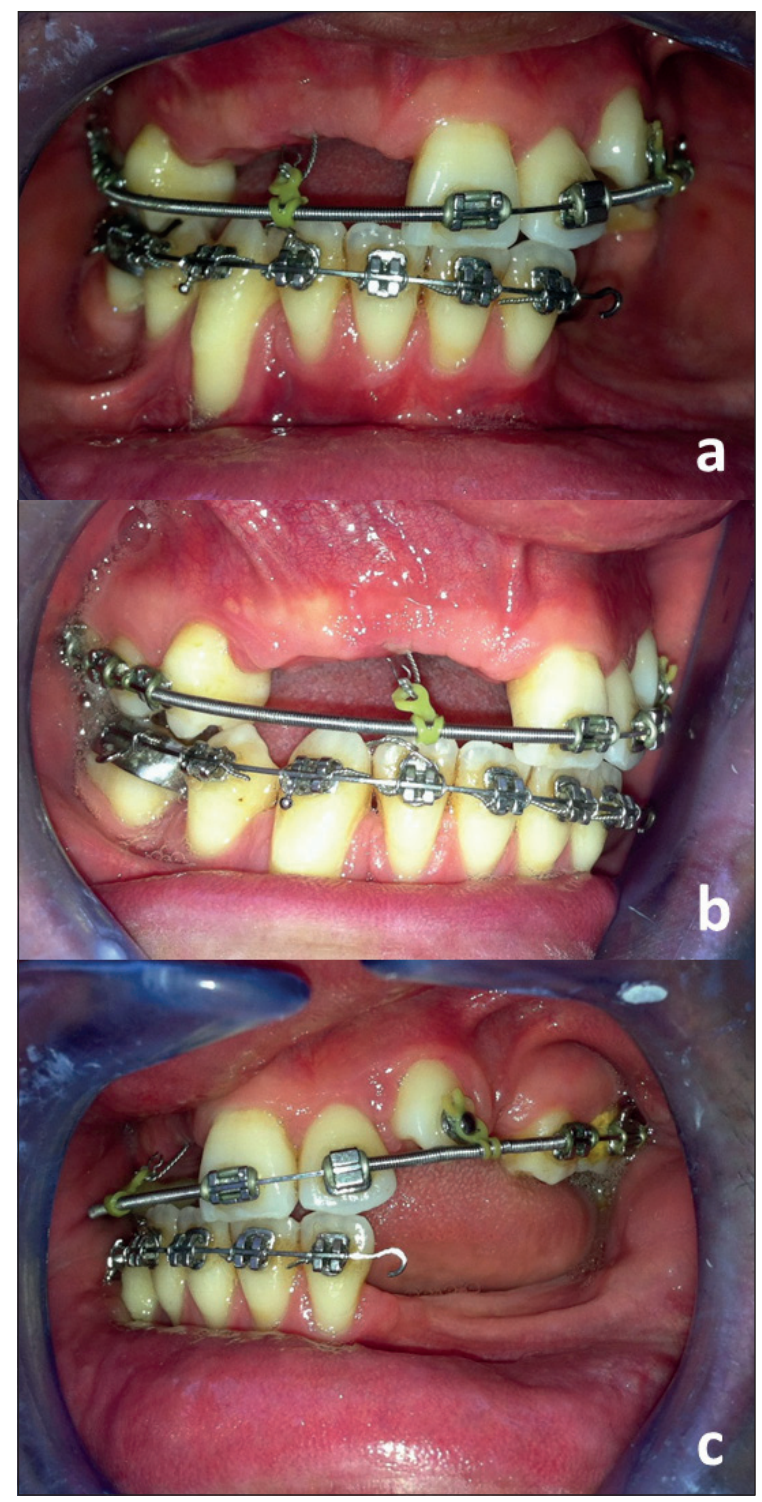

Fig. 10. Photographie montrant la progression après quatre ans du traitement.

Fig. 10. Treatment progress four years post treatment.

Il est à noter que ce patient est toujours en suivi thérapeutique et celui-ci semble satisfaisant du fait de l'éruption spontanée de la 33 bis et la réponse favorable à la traction des 24 bis et 25 bis (Fig. 10).

\section{Discussion}

À l'heure actuelle, il est très difficile de pouvoir établir un plan de traitement global applicable à tous les patients [4].

De ce fait, il doit être individualisé puisque les cas de figure rencontrés sont extrêmement variables $[2,5]$.
L'éventail des propositions thérapeutiques est extrêmement large et de plus en plus audacieux dans ce domaine, allant de l'extraction de toutes les dents et la réhabilitation prothétique (pour des cas légitimes) à la désinclusion orthochirurgicale qui est un traitement élaboré pour des praticiens spécifiquement formés. Entre ces deux extrêmes, il existe toute une gamme de modes d'interventions qui permet de faire un choix précis et individuel en fonction du type d'inclusion rencontrée, et de la précocité du diagnostic et de la prise en charge. Ainsi, plus le patient sera jeune et l'état bucco-dentaire favorable, plus le traitement sera conservateur. Le nombre de dents incluses et surnuméraires, la motivation du patient ou encore les caractéristiques inhérentes à chaque technique seront également déterminants $[3,6]$.

Le traitement ortho-chirurgical a été dans notre cas clinique la solution préférentiellement choisie. En effet, il constitue le mode thérapeutique le plus recherché car donnant les meilleurs résultats. Il consistait à éliminer les obstacles à l'éruption des dents définitives représentées par l'os qui les recouvre, les dents surnuméraires, et à les tracter sur l'arcade $[5,7,8]$.

Cette prise en charge ortho-chirurgicale visera à améliorer et si possible à corriger les troubles masticatoires, phonatoires et esthétiques, ainsi qu'à préserver le patient des troubles médicaux que peut engendrer la présence d'un grand nombre de dents incluses. En fonction de la durée évaluée des interventions, de l'âge et de la coopération du patient, les interventions seront réalisées simultanément au maxillaire et à la mandibule, sous anesthésie locale.

L'abord chirurgical dépend de la localisation des dents définitives dans les bases osseuses, de leurs axes, de leurs morphologies : leurs couronnes devront être capables de réaliser un guide physiologique de l'occlusion, leurs racines devront autoriser les déplacements orthodontiques. Enfin les diamètres coronaires des dents choisies doivent correspondre à l'espace disponible sur l'arcade [9].

Dans certains cas où les dysmorphoses squelettiques sont conséquentes, il arrive que les thérapeutiques chirurgicoorthodontiques ne suffisent pas à parvenir à des résultats satisfaisants.

La chirurgie orthognathique par ostéotomie de Lefort ou par repositionnement mandibulaire peut alors améliorer l'esthétique et la fonction lorsque le traitement s'achève avec une arcade mandibulaire complète associée à une arcade maxillaire rétrusive et à une importante béance antérieure, ou lorsque le traitement est plus tardif et nécessite un fort accroissement de la dimension verticale d'occlusion [10].

Les techniques chirurgico-orthodontiques permettent de traiter la majorité des cas sans prothèse mais il existe toujours une possibilité que les dents ne fassent pas leur éruption.

Dans ces cas d'échec, les thérapeutiques prothétiques pourront être utilisées, mais les prothèses devront être sans cesse modifiées, les risques infectieux sont importants, l'os 
alvéolaire n'est pas maintenu et avec sa disparition s'amplifient les désordres squelettiques ainsi que la perte de rétention.

Bien entendu, des récidives sont à craindre par rapport à un cas plus classique, si de nouvelles dents surnuméraires se forment $[9,11]$.

Cette éventualité doit donc être anticipée par une surveillance radiographique régulière des bases osseuses.

Les difficultés sont accrues dans la DCC par le nombre important d'éléments surnuméraires, un faible potentiel éruptif et davantage de problèmes d'ancrage orthodontiques $[3,12]$.

Ainsi, la prise en charge de ce syndrome est longue et tributaire de l'apparition des dents surnuméraires et de la rapidité d'évolution dentaire et osseuse. Il est donc impossible pour nous, chirurgien-dentistes, de pallier tous les problèmes dentaires rencontrés, en une seule intervention [13]. De plus, les thérapeutiques proposées doivent être souples en fonction de la réponse au traitement, de la compétence et de l'expérience du praticien soignant et de l'engagement du patient [14].

Une prise en charge précoce durant la croissance permet de favoriser un développement transversal et sagittal des bases osseuses [15]. Les patients atteints de DCC ne présentent pas de véritables lésions handicapantes dans leur vie quotidienne. Leur état leur permet d'exercer des métiers qui demandent une dextérité manuelle [16]. L'exemple de notre patient, couturier de profession, en est la parfaite illustration.

\section{Conclusion}

Ce travail a souligné la fréquente complexité de prise en charge de ce type de pathologie dont le traitement peut nécessiter plusieurs étapes successives s'étalant sur des mois voire des années d'où la nécessité d'une véritable approche multidisciplinaire entre le spécialiste en chirurgie dentaire, l'orthodontiste et le chirurgien maxillo-facial pour faire face aux différentes anomalies du massif facial et aux anomalies dentaires et répondre ainsi à la double demande de ce type de patient : fonctionnelle et surtout esthétique.

Il convient de noter qu'une prise en charge précoce durant la croissance permet de favoriser un développement transversal et sagittal des bases osseuses.

Toutefois, l'engagement et l'assiduité du patient jouent un rôle primordial dans la réussite de ce projet thérapeutique.

Conflits d'intérêt : aucun

\section{Références}

1. Petropoulos VC, Balshi TJ, Wolfinger GJ, Balshi SF. Treatment of a patient with cleidocranial dysplasia using a single-stage implant protocol. J Prosthod 2011;20(1):26-31.

2. Cooper SC, Flaitzi CM, Johnston DA. A natural history of cleidocranial dysplasia. Am J Med Genet 2001;104(1):1-6.

3. Golan I, Baumert U, Hrala BP, Mussig D. Early craniofacial signs of cleidocranial dysplasia. Int J Paediatr Dent 2004;14(1):49-53.

4. Cohen MM. Craniofacial abnormalities: clinical and molecular perspectives. Ann Acad Med Singapore 2003;32(2):244-251.

5. Lafargue H, Richard B, Gorry P, Marteau JM, Boileau MJ. La dysplasie cléido-crânienne : attitudes thérapeutiques et recommandations. À propos d'une observation clinique. Act Odontostomatol $2009 ; 247(22): 241-254$.

6. Coffman JA. Runx transcription factors and the developmental balance between cell proliferation and differenciation. Cell Biology International 2003;27(5):315-324.

7. Chen S, Santos L, Wu Y. Altered gene expression in human cleidocranial dysplasia dental pulp cells. Arch Oral Biol 2005;50(2): 227-236.

8. Berg RW, Kurtz KS, Watanabe I, Lambrakos A. Interim prosthetic phase of multidisciplinary management of cleidocranial dysplasia: "the Bronx Approach". J Prosthodont 2011;20(2):20-25.

9. Roberts $T$, Stephen L, Beighton P. Cleidocranial dysplasia: a review of the dental, historical, and practical implications with an overview of the South African experience. Oral Surg Oral Med Oral Pathol Oral Radiol 2013;1(15):46-55.

10. Dalessandri D, Laffranchi L, Tonni I, Zotti F, Piancinon MG, Pagnelli C, et al. Advantages of cone beam computed tomography (CBCT) in the orthodontic treatment planning of cleidocranial dysplasia patients: a case report. Head Face Med 2011;27(7):6-9.

11. Libersa $P$, Roze D, Libersa JC. Hyperodontie et dysplasie cléidocrânienne. À propos d'un cas. J Dent Québec 2003;40(12): 125-129.

12. Lossdorfer S, Abou jamra B, Rath-Deschner B, Götz W. The role of periodontal ligament cells in delayed tooth eruption in patients with cleidocranial dysostosis. J Orofac Orthop 2009; 70(6):495-510.

13. Manjunath K, Kavita B, Saraswathi TR, Sivapathasundharam B, Manikandhan R. Cementum analysis in cleidocranial dysostosis. Indian J Dent Res 2008;19(3):253-256.

14. Golan I, Waldeck A, Baumert U, Strutz J, Müssig D. Anomalies of the skull in cleidocranial dysplasia. An otorhinolaryngological and craniofacial radiological study. HNO 2004;52(12):1061-1066.

15. Molla M, Baillaul I, Verloes A, Berdal A. The genetic basis of inherited anomalies of the teeth. Part 1: clinical and molecular aspects of non-syndromic dental disorders. Eur J Med Genet 2008;51(4):273-91.

16. Ryoo HM, Kang HY, Lee SK, Lee KE, Kim JW. RUNX2 mutations in cleidocranial dysplasia patients. Oral Dis 2010;16(1):55-60. 